Cahiers $d u$ MONDE RUSSE

\section{Cahiers du monde russe}

Russie - Empire russe - Union soviétique et États indépendants

48/2-3 | 2007

Les résonances de 1905

\title{
La révolution de 1905 dans l'opinion républicaine française
}

\section{Wladimir Berelowitch}

\section{OpenEdition \\ Journals}

Édition électronique

URL : https://journals.openedition.org/monderusse/9012

DOI : 10.4000/monderusse.9012

ISSN : $1777-5388$

Éditeur

Éditions de l'EHESS

\section{Édition imprimée}

Date de publication : 15 avril 2007

Pagination : 379-392

ISBN : 978-2-7132-2147-7

ISSN : $1252-6576$

Référence électronique

Wladimir Berelowitch, "La révolution de 1905 dans l'opinion républicaine française », Cahiers du monde russe [En ligne], 48/2-3 | 2007, mis en ligne le 01 janvier 2007, consulté le 04 septembre 2022 URL : http://journals.openedition.org/monderusse/9012; DOI : https://doi.org/10.4000/monderusse. 9012 
chercher : repérer : avancer

Cet article est disponible en ligne à l'adresse :

http://www.cairn.info/article.php?ID REVUE=CMR\&ID NUMPUBLIE=CMR 482\&ID ARTICLE=CMR 4820379

\title{
La révolution de 1905 dans l'opinion républicaine française
}

\author{
par WLADIMIR BERELOWITCH
}

\section{Editions de l'EHESS | Cahiers du monde russe}

2007/2-3 - Vol 48

ISSN 1252-6576 | ISBN 9782713221477 | pages 379 à 392

Pour citer cet article :

-BERELOWITCH ., La révolution de 1905 dans l'opinion républicaine française, Cahiers du monde russe 2007/ 2-3, Vol 48, p. 379-392.

Distribution électronique Cairn pour les Editions de l'EHESS.

(C) Editions de l'EHESS. Tous droits réservés pour tous pays.

La reproduction ou représentation de cet article, notamment par photocopie, n'est autorisée que dans les limites des conditions générales d'utilisation du site ou, le cas échéant, des conditions générales de la licence souscrite par votre établissement. Toute autre reproduction ou représentation, en tout ou partie, sous quelque forme et de quelque manière que ce soit, est interdite sauf accord préalable et écrit de l'éditeur, en dehors des cas prévus par la législation en vigueur en France. Il est précisé que son stockage dans une base de données est également interdit. 


\section{LA RÉVOLUTION DE 1905 DANS L'OPINION RÉPUBLICAINE FRANÇAISE}

On connaît assez bien, dans ses grandes lignes, l'évolution des relations francorusses au début du $\mathrm{Xx}^{\mathrm{e}}$ siècle, notamment sous l'angle diplomatique et financier. Comme on le sait ${ }^{1}$, la Guerre russo-japonaise et la révolution de 1905 mirent l'alliance franco-russe à rude épreuve sous trois aspects essentiels. D'abord, la situation financière très difficile de la Russie, par suite de la guerre, l'obligea à rechercher des emprunts (en 1904, puis en 1906) en grande urgence, ce qui la plaçait dans une position de faiblesse et de dépendance vis-à-vis de son alliée. Ensuite, les revers militaires et les troubles révolutionnaires qui frappaient l'Empire russe suscitèrent de sérieux doutes en France, y compris au niveau gouvernemental, concernant la fiabilité militaire et politique de son alliée de l'Est. Cette perte de crédit se traduisit du reste par une chute des valeurs russes en bourse, une chute que les efforts conjugués des autorités françaises et russes n'enrayèrent qu'à grand-peine. Enfin, cette nouvelle méfiance par rapport à la puissance russe ne se traduisit pas seulement par des mouvements de l'opinion défavorables à la monarchie russe. Elle s'accompagna d'exigences actives formulées par cette même opinion et des demandes discrètes au niveau gouvernemental, poussant le pouvoir russe à la paix avec le Japon et à des réformes libérales.

Les années 1904-1907 jouèrent donc un rôle décisif dans le sort de l'alliance franco-russe. Si, d'une part et pour l'essentiel, la politique des deux puissances

1. Voir, notamment, sur les aspects financiers de l'Alliance, les travaux de René Girault, Emprunts russes et investissements français en Russie, 1887-1914 rééd., P. : IGPDE, 1999 et de Boris V. Anan'ič, Rossija i meždunarodnyj kapital [La Russie et le Capital international], L. : Nauka, 1970. Voir aussi le recueil Russkie finansy i evropejskaja birža v 1904-06 gg. [Les finances russes et la bourse européenne en 1904-1906], B.A. Romanov, ed., M.-L., 1926 et la thèse inédite de James William Long, The Economics of the Franco-Russian Alliance, 19041906, University of Wisconsin, 1968. L'ouvrage d'Anne Hogenhuis-Seliverstoff, Une alliance franco-russe : la France, la Russie et l'Europe au tournant du siècle dernier, Bruxelles : Bruylant - LGD, 1998, traite de la période précédente ; voir, cependant, un aperçu des années 1905-1906 p. 182-183. 
demeura inchangée malgré un refroidissement assez sensible des sentiments enthousiastes de part et d'autre, elle fut paradoxalement infléchie dans le sens d'un resserrement, tout au moins du côté du gouvernement russe qui fut obligé de soutenir son allié français contre l'Allemagne dans l'affaire marocaine, à la conférence d'Algésiras, puis de se rapprocher de l'Angleterre jusqu'à la formation de la Triple Entente, ce qui détermina le cours de la politique internationale jusqu'à la Grande Guerre. Par ailleurs, la perspective, plus ou moins réaliste, d'une évolution de la Russie vers une démocratie parlementaire accusa les clivages et les interrogations qui travaillaient déjà l'opinion républicaine française - des socialistes aux radicaux, d'autant plus que désormais, il se trouvait dans l'opposition russe des interlocuteurs assez autorisés pour influencer cette opinion.

C'est vers ce dernier aspect que nous voudrions nous tourner. Il est mal connu, exception faite des travaux de Gilles Candar sur les socialistes français ${ }^{2}$, de quelques travaux sur les réactions de l'opinion française de façon générale ${ }^{3}$ et des révélations des archives russes sur la fameuse «vénalité de la presse française ${ }^{4}$. Même ce dernier point mériterait d'être affiné. À ce jour, nous n'avons pas et n'aurons probablement jamais une idée exacte de l'efficacité réelle de l'argent versé par le gouvernement russe, avec la complicité de son homologue français, à des journaux comme Le Petit Parisien ou Le Matin. Ces sommes considérables 2500000 francs de 1904 à 1906 - servirent surtout à financer des publicités sur les emprunts russes et il semble qu'il y ait eu entre certains journaux, comme les deux derniers cités, et les agents russes, un marché de dupes qui ne profita guère à l'image de marque de la Russie. En effet, la logique de l'information interdisait à une presse qui recherchait la sensation de minimiser des événements aussi énormes que le Dimanche rouge, le désastre de Tsushima ou la grève générale d'octobre 1905. Même dans le cas de journaux libéraux comme le Journal des débats ou Le Temps, à plus forte raison Le Figaro, plus à droite, il n'est pas sûr que ce fût l'argent français qui eût suscité une méfiance à l'égard de la révolution russe, sentiment qu'ils pouvaient éprouver sans aucun encouragement financier,

2. Nous laisserons pratiquement de côté les réactions socialistes, sauf lorsqu'elles s'inscrivaient dans un contexte plus large, car elles sont traitées ici par Gilles Candar, ainsi que dans son article : «Les socialistes français et la Révolution russe de 1905 », Bulletin de la Société des études jaurésiennes, $\mathrm{n}^{\circ} 131$, janvier-mars 1994, p. 3-10; voir aussi son ouvrage Jean Longuet, 1876-1938. Un internationaliste à l'épreuve de l'histoire, Rennes : Presses Universitaires de Rennes, 2007.

3. Voir, notamment, Denise Eeckaute, «1905 dans la presse française et anglaise (étude comparée) », in François-Xavier Coquin et Céline Gervais-Francelle, éds., 1905, la première révolution russe, actes du colloque de 1985, P. : Institut d'Etudes slaves - Sorbonne, 1986, p. 451-466; René Girault, «La révolution russe de 1905 d'après quelques témoignages français », La Revue historique, 230 (1), 1963, p. 97-120 ; P. Truchot, La presse et l'opinion publique française face à la révolution russe de 1905, mémoire de maîtrise, Université de Nantes, 2000 et, surtout, la thèse inédite de James B. Northcutt, Contemporary French evaluations of the Russian Revolution of 1905, Berkeley : University of California, 1974.

4. Krasnyj arhiv, 1925, n’ 3 (10), traduit par Boris Souvarine dans L'Humanité communiste (en ouvrage séparé : Arthur Raffalovitch, L’abominable vénalité de la presse française, P. : Librairie du travail, 1931). Voir une synthèse sur cette question par James Long, « The Russian manipulation of the French press, 1904-1906 », Slavic Review, vol. 31 (2), June 1972, p. 343-54 
ce qui ne les empêchait d'ailleurs pas de couvrir l'actualité russe sans beaucoup de complaisance pour le tsar ${ }^{5}$.

Nous nous bornerons ici à esquisser quelques directions de recherche concernant les réactions de l'opinion « de gauche » (républicaine) à la révolution russe, en nous fondant principalement sur une lecture de la presse de l'époque.

Premier constat, ces événements suscitèrent un fort émoi à la gauche de l'échiquier politique. Il s'ensuivit quelques joutes parlementaires, au cours desquelles le ministre des Affaires étrangères Delcassé eut maille à partir, face aux socialistes, pour défendre la politique officielle du gouvernement Rouvier (le premier gouvernement Rouvier qui venait de se former au moment du Dimanche rouge), politique qu'on pourrait résumer ainsi : poursuite de l'alliance franco-russe y compris par de nouveaux emprunts, et stricte non-ingérence dans les affaires intérieures russes. Les réactions du parti socialiste et des autres organisations d'extrême-gauche furent, évidemment, extrêmement vives et conformes à la tradition anti-tsariste de la gauche française. Par contre, le parti radical demeura dans l'ensemble très discret, que ce fût à la Chambre ou dans ses congrès annuels. Le congrès de 1905 (le Cinquième) se contenta de saluer les efforts du président Roosevelt pour obtenir une paix russo-japonaise, ce qui ne le démarquait guère de la position de neutralité affichée par Delcassé. Mais l'attitude du parti évolua quelque peu en 1906, sous la présidence de Camille Pelletan. Le 19 octobre 1906, à la veille de la chute du gouvernement Sarrien et trois mois après la dissolution de la Première Douma, le Congrès de Lille (le Sixième) votait une résolution proposée par la commission aux affaires extérieures et coloniales, qui contenait des critiques à peine voilées du gouvernement russe :

$1^{\circ}$ Le Congrès flétrit avec indignation les massacres organisés qui ensanglantent la Russie.

Croit accomplir son devoir d'amitié envers la nation alliée en lui rappelant, dans un esprit de pacification et dans un sentiment d'humanité, que selon les termes de la Déclaration séculaire qui a assuré ses libertés et ses progrès, «l'oubli et le mépris des droits naturels de l'homme sont les seules causes des malheurs du monde» et que «la loi doit être l'expression de la volonté générale ». Proteste, en conséquence, contre la dissolution de la Douma et salue sa réunion prochaine aux cris de «Vive la Douma! »

$2^{\circ}$ Le Congrès

Considérant que les derniers gouvernements qui se sont succédé au pouvoir ont affirmé leur souci de ne pas contracter d'emprunts nouveaux, ils ont, à plusieurs reprises, favorisé l'émission, en France, d'emprunts contractés par certains gouvernements étrangers.

5. James Long (op. cit.) conclut son article par l'idée que l'argent versé à la presse française valut au gouvernement russe d'engranger 2 milliards de francs d'emprunts de 1904 à 1906, car une bonne partie de la presse française sous-informa systématiquement ses lecteurs. Cette conclusion peut s'appliquer dans une certaine mesure à la période qui précéda le Dimanche rouge et les désastres de Moukden et de Tsushima, mais nous semble beaucoup trop simpliste si on se tourne vers la période suivante. L'auteur sous-estime visiblement les impératifs qui guidaient la grande presse d'information de cette époque et ne fait que reprendre l'image qu'en ont laissée ses pourfendeurs d'extrême-gauche. 
Proteste contre cette politique financière, anti-démocratique et antinationale, dangereuse pour la paix du monde, qui compromet la fortune publique, pour les plus grands profits de la haute finance, et alimente à l'étranger les forces de réaction, avec l'épargne du pays républicain, au détriment des œuvres françaises. $[\ldots]^{6}$

Bien que sous une forme modérée, la résolution reprenait, en fait, l'essentiel des critiques de la gauche, puisqu'elle stigmatisait la répression, les pogroms dirigés contre les juifs et les Arméniens, la dissolution de la Douma et l'utilisation des emprunts à des fins peu recommandables, puisque la guerre contre le Japon, puis l'usage de la troupe contre la population russe avaient prouvé qu'ils n'avaient servi ni la paix, ni les libertés. Cette critique, toutefois, n'alla jamais jusqu'à remettre en cause les gouvernements Rouvier, Sarrien (de mars à octobre 1906), puis, à partir du 25 octobre, Clemenceau. En dehors des évolutions internes au parti radical, et sans doute de l'influence personnelle de Pelletan qui sympathisait avec la révolution russe ${ }^{7}$ comme il sympathisait avec les mouvements sociaux français, il faut surtout voir dans cette désapprobation une réaction quasi instinctive des républicains à la dissolution de la Douma, venant d'un pays comme la France, où l'intégrité et la stabilité du pouvoir législatif étaient des conquêtes récentes.

Entre les socialistes et les radicaux centristes, nous trouvons Clemenceau et la mouvance qu'on peut qualifier de radicaux de gauche et qui, avant l'accession du « Tigre » au gouvernement Sarrien comme ministre de l'Intérieur, consacra une très grande, et une très virulente attention aux événements russes. De janvier 1905 à mars 1906, il ne se passa pas une semaine, voire, parfois, au plus fort des événements en Russie, un jour, sans que, comme il l'avait fait au début de l'Affaire Dreyfus dans ses éditoriaux à L'Aurore, Clemenceau ne prît sa plume redoutable pour la diriger cette fois contre le gouvernement russe et la politique étrangère, à son gré trop indulgente pour le tsar, de sa bête noire de Delcassé.

La position de Clemenceau était simple et cohérente ${ }^{8}$ : la démocratie française pouvait s'allier à la monarchie absolue russe tant que ses intérêts y trouvaient leur compte. Mais cela ne devait en aucun cas la conduire à se renier en blanchissant le gouvernement du tsar de ses exactions, ni à s'aveugler sur son avenir plus que compromis, ni, enfin et surtout, à se mettre à la remorque d'une politique extérieure irresponsable et délictueuse qui pouvait conduire l'Europe à la guerre puisqu'elle mettait en cause les intérêts anglais. Son devoir était d'aider la Russie à évoluer vers

6. Parti républicain radical et radical-socialiste. Sixième congrès annuel, P. : Comité exécutif, 1906, p. 139.

7. Il fut assez proche de la Société des amis du peuple russe et des peuples opprimés, qui publia son discours du 5 décembre 1905, La Révolution en Russie, P., décembre 1905.

8. Hormis l'ouvrage de David Robin Watson, Georges Clemenceau. A political biography, Londres : Eyre Methuen, 1974, où on trouve une discussion de ce problème, les biographies de Clemenceau n'accordent que très peu ou guère de place à son attitude devant la révolution russe, sujet qui mériterait une étude détaillée à lui seul. Voir, par exemple, Jean-Baptiste Duroselle, Clemenceau, P. : Fayard, 1988, p. 488-490 ; rien chez Philippe Erlanger, Clemenceau, P. : Grasset, 1968, ni chez Michel Winock, Clemenceau, P. : Perrin, 2007. 
un gouvernement représentatif. Principes et intérêts se rejoignaient donc chez Clemenceau dans une politique qui, dans l'idéal, aurait dû conjuguer prudence et volonté.

Ainsi, dans L'Aurore du 31 janvier 1905, soit neuf jours après le Dimanche rouge : L'alliance franco-russe « que tout semblait contre-indiquer dans l'histoire, fut, pour les deux parties, l'effet d'une nécessité inéluctable». Mais : « Ces faits [le massacre] rien ne louera M. Delcassé d'avoir cherché à les excuser, à les justifier devant l'universelle réprobation du genre humain. [...] Louis XIV et Cromwell ont pu se rapprocher tout en restant eux-mêmes. La démocratie française a pu et peut encore débattre des intérêts internationaux du tsar. Mais M. Delcassé nous a fait le plus sanglant outrage quand il ose nous demander de manifester d'autres sentiments que ceux d'une démocratie. Aussi longtemps que les peuples gémiront sous le talon de l'oppresseur, le cœur de la démocratie française s'élancera vers les opprimés. [...] La guerre russo-japonaise, les massacres à Saint-Pétersbourg ont révélé l'extraordinaire faiblesse matérielle et morale de notre allié. »

Le 14 mars 1905, après le désastre de Moukden : « Trop longtemps nous avons accepté un rôle de passivité dans l'alliance jusqu'à méconnaître nos intérêts les plus manifestes [...]. Le temps n'est-il pas venu pour nous d'une intervention active auprès de notre alliée en vue de sauver de la mise française dans l'alliance ce qui peut encore en être sauvé ? Le premier besoin de la Russie est de mettre fin à la guerre pour se donner un régime de paix civile par le moyen d'un ordre de justice et de liberté. Notre concours lui sera précieux pour se refaire. Elle peut en quelques années de patient labeur rétablir ses forces épuisées et peser de nouveau d'un grand poids dans les affaires de l'Occident. L'heure est venue pour son gouvernement de dire si cette destinée le tente ou s'il choisit d'aggraver le désastre de ses armées de terre et de mer par les bouleversements de la Révolution. » Ou encore, le 20 juillet 1905, dans un éditorial intitulé «La double paix », dans lequel Clemenceau lie étroitement politique intérieure et extérieure : «L'autocratie est-elle en état de soutenir la double guerre contre le Japon et contre ses propres sujets ? L'expérience a prononcé dans les deux domaines. Pour la double paix le tsar se voit mis en demeure de céder au Japon comme de céder à la Russie. »

Mais si la France doit aider la Russie à transformer la double guerre en double paix, elle ne doit pas pour autant se bercer d'illusions sur le chaos russe; le 26 décembre 1905, soit pendant l'insurrection de Moscou, Clemenceau polémique avec les socialistes français, tout en stigmatisant le gouvernement russe :

Villes et campagnes, la révolte surgit de toutes parts. [...] La grève systématique $^{9}$ refuse à la communauté une participation de vie, dont les maîtres de l'ordre social n'ont su faire qu'une participation de misères et d'inépuisables douleurs. Les paysans se ruent sur les châteaux et sur les bandes révolutionnaires indifféremment, faute de comprendre la cause de leurs maux. Les soldats

9. Comme beaucoup d'autres gens de gauche, Clemenceau évite l'expression « grève générale » à cause de ses consonances anarcho-syndicalistes. Ailleurs (L'Aurore du 21 mars 1905), et à propos de la grève scolaire en Pologne, il emploie aussi l'expression de « grève universelle ». 
tirent au hasard contre les uns ou les autres, quelquefois même, sans savoir pourquoi, refusent de tirer. Cependant à Moscou, dans la vieille capitale de la Russie, on se bat à coups de canon. [...] C'est le « libéralisme » de M. Witte qui est à l'œuvre. [...] J'admire les doctrinaires de la révolution qui tirent, de ces spectacles, avec superbe, des prédictions d'universel bonheur. Victoire ou défaite de la répression $[\ldots]$, je ne puis qu'entrevoir, pour le grand peuple qui a le plus souffert de la domination sans frein, une suite nouvelle de malheurs. [...] Je suis trop ambitieux de révolution vraie pour attendre de la simplicité d'un coup de force populaire le succès durable d'une idée. La violence de tous est bonne, à certaines heures, pour contenir la violence de quelques-uns. Elle ne peut suffire à fonder. [...] Nous qui savons quelle tâche ingrate demeure pour qui veut libérer l'homme des chaînes de son ignorance, nous à qui la défaite de la révolution, au Deux-Décembre ${ }^{10}$, impose aujourd'hui de défendre la France des entreprises d'une domination militaire et de sauvegarder et de développer la révolution de nos aïeux non plus dans l'enthousiasme et dans la violence, mais dans le calme et la méthode d'une réflexion mûrie, il nous appartient de combattre à notre tour pour la cause commune en donnant l'exemple d'un victorieux effort pour nous ordonner, nous discipliner, allier harmonieusement et l'impulsion de la volonté et la règle de modération (qui est encore une part d'énergie) en vue de l'efficacité supérieure de l'action. Il est plus facile de détruire le despotisme que de l'empêcher de renaître sous une forme nouvelle, plus facile de supprimer un tyran que de fonder, d'organiser la liberté.

L'antipathie de Clemenceau pour le gouvernement russe était telle qu'Aleksandr Nelidov, l'ambassadeur de Russie, mit en garde le président Fallières quand Sarrien fit entrer le « Tigre » dans son gouvernement, « Tigre » qui était opposé jusqu'en mars 1906 à l'octroi d'un nouvel emprunt sans conditions à la Russie11. Comme on le sait, une fois au gouvernement et bien avant le coup d'État de Stolypin qui n'allait rien changer à l'affaire, Clemenceau allait suivre une stricte politique d'intérêt.

Nous avons longuement cité Clemenceau parce que ses éditoriaux, vigoureux et incisifs, rendent assez bien compte, tant par l'attention portée aux événements russes que par leur interprétation, de l'ensemble de l'opinion républicaine, socialistes non compris.

Opinion qui s'exprima avant tout dans la presse. De façon générale, les événements de Russie firent «la une » des journaux aux moments les plus forts de la révolution : après le « Dimanche rouge » en janvier-février 1905, évidemment, puis à plusieurs reprises au cours de l'année 1905 (en mai lors du congrès des zemstva, en juin autour de la mutinerie du cuirassé «Potemkin»...), et avec un second

10. Clemenceau songe évidemment au coup d'État de Louis-Napoléon Bonaparte le 2 décembre 1851, à une époque où le souvenir du boulangisme reste encore frais.

11. Comme il l'écrit dans L'Aurore du 30 janvier 1906 : « [...] on lui a payé sa guerre en Mandchourie [...] Il nous reste à lui assurer sa victoire sur ses propres sujets. C'est ce que nous nous sommes attachés scrupuleusement à faire jusqu'à ce jour. » Sur cette question, voir Pierre Renouvin, «L'emprunt russe d'avril 1906 en France », Schweizer Beiträge zur Allgemeinen Geschichte, 18-19, 1960-61, p. 507-515 ; également : David Robin Watson, Georges Clemenceau..., p. 217. 
apogée d'octobre à décembre1905 ; en 1906-1907, les premières pages des journaux furent surtout alimentées par l'actualité des trois doumas successives.

Mais cette attention de la presse ne surgissait pas dans un désert. D'une part, la guerre russo-japonaise, hautement inquiétante pour l'allié français de la Russie, était largement reflétée dans la presse depuis janvier 1904. Du reste, les journaux présentaient souvent les progrès de la révolution parallèlement aux événements relatifs à cette guerre et souvent dans les mêmes pages, de sorte que, on l'a bien vu dans le cas de Clemenceau, il n'était guère possible de les distinguer. D'autre part, le congrès et la fronde des zemstva en novembre 1904, puis les troubles de Bakou et enfin les premiers brûlots de Petrograd, avaient déjà trouvé des oreilles attentives à Paris. C'est ainsi que dans L'Aurore, l'actualité russe s'installa en première page le 12 décembre 1904, sous le titre «Agitation en Russie». Elle ne devait plus la quitter. Le 20 janvier 1905, le journal se mettait en attente d'un grand bouleversement en Russie avec ce titre suggestif : «Révolution ? ». Et le 23 janvier, soit au lendemain du «Dimanche rouge», le même titre revenait, mais cette fois affirmatif : «Révolution! »

Deux groupes de journaux, particulièrement, furent sensibles à l'actualité russe : d'abord la presse marquée à gauche (presse socialiste, presse radicale de gauche comme L'Aurore, presse influencée par le radicalisme de gauche comme Le Radical, La Lanterne et d'autres journaux) et ensuite la grande presse d'information (Le Petit Parisien, Le Matin, Le Journal, L'Écho de Paris). L'explication paraît simple : dans le premier groupe, c'est l'opinion l'anti-tsariste, dans le second, comme on l'a vu, le souci d'informer les lecteurs qui furent déterminants. Par exemple, si Le Matin et L'Écho de Paris du 23 janvier 1905 s'interrogent sur l'événement (émeute ou révolution ?) ils n'éprouvent guère de doute sur deux constats : c'est de toute façon un bain de sang dont le gouvernement russe est responsable, et c'est la première fois que le peuple russe se soulève contre sa misère. Plus tard, le correspondant du Matin en Russie, Gaston Leroux, ne va pas cacher ses sympathies pour le camp de la révolution. Quant au Petit Parisien (29 janvier), il souligne le caractère universel, réellement populaire de ce soulèvement et prophétise une rébellion généralisée des classes laborieuses qui nécessitera ( 23 janvier) un changement institutionnel majeur en Russie.

Les journaux libéraux du centre à plus faibles tirages, comme Le Temps ou le Journal des débats, ainsi que Le Figaro, furent nettement plus discrets.

Le Journal des débats, où Alcide Ebray couvre les événements russes, est réformiste lorsqu'il s'agit de Russie, et par conséquent appuie les transformations du régime monarchique qui peuvent aller, même à tout petits pas comme la Douma de Bulygin, dans le sens d'un gouvernement représentatif. Mais cela n'empêche pas le même Ebray de tirer la sonnette d'alarme, comme s'il doutait de cette perspective rassurante : «C'est ainsi qu'on commence les révolutions, beaucoup plus qu'on ne prépare les réformes. Mais la Russie n'a expérimenté ni les unes, ni les autres et il semble bien que l'opinion ne s'y soit pas rendu compte de la gravité de la situation » (le 22 janvier 1905). Et Anatole Leroy-Beaulieu, qui y écrit régulièrement, prend soin de se démarquer des vues trop étroites de ce journal en écrivant, le 19 novembre 1905, soit après le manifeste d'Octobre : «S'il y a lieu de s'affliger 
des troubles et des massacres qui ont ensanglanté la Russie, on ne saurait en être étonné. Ce qui se passe dans le grand empire n'est rien [de] moins qu'une révolution, une révolution qui, à travers des péripéties diverses, ne sera peut-être ni moins longue, ni moins tragique que la nôtre, bien que, à l'heure présente, on puisse encore espérer qu'elle s'opérera sans le renversement du trône et de la dynastie. Ce que serait une révolution russe, si le pouvoir central venait à disparaître, si l'empereur ne survivait pas à l'autocratie, l'anarchie de ces dernières semaines nous en donne comme un avant-goût. »

La lecture du Temps ne laisse guère de place à la perspective d'une révolution victorieuse en Russie, ce terme même apparaissant fort peu. Sous la rubrique générale « Bulletin de l'étranger », on voit paraître en effet des titres plutôt rassurants et neutres, tels que «Les réformes en Russie » (le 29 janvier 1905), « La France et l'alliance russe » (le 2 février), «Les événements de Russie et l'alliance francorusse» (le 4 février). Mais Le Temps observe des limites raisonnables dans sa prudence et juge par exemple, dans le même numéro du 29 janvier, que le massacre du Dimanche rouge est injustifiable. En juillet 1906, après la dissolution de la Première Douma, nous observons une sorte de crescendo dans la réprobation et l'inquiétude : le 23 juillet 1906, l'éditorialiste anonyme accuse les conseillers de Nicolas II qui «viennent de lui faire violer la promesse qu'il avait souscrite en convoquant la Douma. Car la Douma [...] n'a pas justifié la mesure dont elle est l'objet. Et c'est pour cela que l'avenir est sombre. Le peuple avait confiance dans l'appel que l'empereur lui avait adressé. Quand il verra revenir les membres de l'Assemblée dissoute par oukase impérial, que deviendra cette confiance ? Peutêtre espère-t-on qu'une Chambre élue autrement sera plus facile à mener. Cet espoir est d'une évidente vanité. Peut-être compte-t-on qu'on pourra éviter même la réunion d'une nouvelle Douma. Mais au prix de quelle répression ? Et d'ailleurs avec quoi réprimer et au nom de quoi ? Pour avoir manqué de courage dans la pratique d'un régime nouveau et difficile, le gouvernement impérial vient d'engager le pays dans une crise dont on ne peut ni mesurer les dangers ni apercevoir le terme. » Le 24 juillet : « ...à voir le libéralisme du gouvernement russe se projeter toujours dans l'avenir, on se prend à douter qu'il ait jamais été sincère. » Enfin, le 25 juillet : «...pour que la force prime le droit, il faut tout au moins qu'elle soit disciplinée. Et qui oserait garantir que l'armée russe menée par la bureaucratie soit de taille à mater un peuple qui se lève contre l'arbitraire ? »

En dehors de la presse, les événements suscitèrent une assez forte mobilisation de l'opinion de gauche. Comment caractériser les formes de cette mobilisation, ainsi que son spectre politique ? De façon générale, et si l'on se fonde d'une part sur la presse, et d'autre part, sur les rapports de police conservés aux Archives nationales de France sous le titre «manifestations anti-tsaristes » ${ }^{12}$, on peut dire que celles-ci se sont cristallisées autour de deux moments : le premier est le mois qui 
suivit le Dimanche rouge. Le second fut la commémoration de cet événement, qui coïncida, à quelques semaines près, avec la répression de l'insurrection de décembre 1905 à Moscou. La forme que prit cette mobilisation fut surtout le meeting, même s'il y eut quelques petites manifestations ${ }^{13}$. Le plus grand se déroula le 30 janvier 1905, soit huit jours après le Dimanche rouge, dans la salle du Tivoli Vaux Hall à Paris. Il réunit au moins 6000 personnes, qui écoutèrent une lettre de Kropotkin et entendirent les discours électrisés de Vaillant, élu président du meeting, Il’ja Rubanovič (Ilya Roubanovitch dans son orthographe française) représentant des socialistes-révolutionnaires russes, les socialistes Jean Allemane, Bracke, Jaurès, Renaudel, le syndicaliste Yvetot, Francis de Pressensé, président de la Ligue des droits de l'Homme... ${ }^{14}$ Dans de nombreuses villes de province pratiquement toutes les grandes villes et beaucoup de villes moyennes ${ }^{15}-$ des meetings réunirent jusqu'à plusieurs centaines de personnes chacun. Une affichette, tirée à 400000 exemplaires et signée par des membres de l'Association internationale antimilitariste ainsi que des syndicalistes (CGT et Jeunesse syndicaliste), dont Almereyda, Malato, Yvetot, Albert Lévy, Monatte, etc., et qui appelait le peuple français à rompre toute relation avec la Russie officielle, fit pratiquement le tour de la France, puisque la police signala sa présence dans de nombreuses villes. Les organisateurs étaient généralement des socialistes, mais on trouve aussi des groupes de Libre pensée, la Ligue des droits de l'Homme, des groupes polonais et russes. Enfin il faut mentionner la Société des amis du peuple russe et des peuples opprimés déjà mentionnée, constituée dès les premiers jours qui suivirent le Dimanche rouge, principalement parisienne, mais qui parvint aussi à mobiliser 1200 personnes à Lyon. Présidée par Anatole France, alors socialiste, elle se situait dans une mouvance qui allait des socialistes aux radicaux, collabora étroitement avec la Ligue des droits de l'Homme et comptait aussi des personnalités non politiques. On trouve, parmi ses membres, Madame Ménard-Dorian, la veuve d'Émile Zola, Pierre Quillard, Jean Psichari, Octave Mirbeau, Paul et Victor Margueritte, Francis de Pressensé, Georges Clemenceau, Pelletan, Ferdinand Buisson, Charles Seignobos, Lucien Herr, Pierre et Marie Curie, Marcelin Berthelot, Camille Flammarion, Paul Painlevé...

Autre forme d'action, la souscription. L'Humanité, L'Aurore, Le Radical et d'autres journaux ouvrirent leurs colonnes aux listes de souscripteurs, principalement à ceux qui répondirent à l'appel du Comité universitaire de protestation contre les massacres de Saint-Pétersbourg.

Précisément, l'examen de ces listes ainsi que des personnalités qui prenaient part aux meetings et aux autres actions de propagande permet de dégager quelques

13. Par exemple, une manifestation d'étudiants parisiens sur le boulevard Saint-Michel le 23 janvier 1905 (voir L'Humanité du 24 janvier).

14. Voir L'Humanité du 28 janvier, ainsi que L'Aurore et d'autres journaux.

15. En relevant seulement les rapports de police, certainement incomplets, on en comptabilise une cinquantaine. 
tendances ${ }^{16}$. Si l'extrême-gauche est dominante parce que la plus active et sans doute la plus nombreuse, le spectre politique anti-tsariste mord largement sur les milieux républicains, de sorte qu'on peut parler d'un continuum entre les deux. Parmi les plus actifs, on peut relever Anatole France qui, déjà mobilisé contre la guerre russo-japonaise ${ }^{17}$, multiplia meetings et prises de position, Jean Jaurès, Octave Mirbeau, Francis de Pressensé, Charles Malato et Yvetot, déjà cités, Ferdinand Buisson, Gabriel Séailles, Édouard Vaillant, Eugène Fournière (directeur de la Revue socialiste) des figures comme Élisée Reclus (qui allait mourir en juillet 1905), Séverine... D'autres écrivains, outre ceux que nous avons cités, et des artistes renommés furent mobilisés de façon plus éphémère pour des appels à la population. Ainsi Alfred Capus, Jules Renard, Tristan Bernard, Rosny aîné, Catulle Mendès, Paul Hervieu, Paul Adam, Maeterlinck, Henriette Psichari-Renan (la fille d'Ernest Renan), la veuve d'Émile Zola, Marquet, Matisse, Signac, Monet, Vallotton, Vuillard, Rodin, Maillol...

Plus discrète, mais néanmoins présente y compris dans les prises de parole, on trouve la «République des professeurs ». Les plus virulents furent l'historien Charles Seignobos (républicain radical), Jean Psichari (École pratique des Hautes Études), Paul Painlevé (École Normale Supérieure), Ferdinand Buisson. Mais on peut citer aussi des universitaires parisiens dont beaucoup se regroupèrent dans le Comité universitaire de protestation évoqué ci-dessus, comme Alphonse Aulard (surtout lui), Louis Havet, Lévi-Bruhl, Sylvain Lévi, Jean Perrin, Paul Langevin, Pierre et Marie Curie, Charles Richet, Charles Lyon-Caen, Charles Gide, Jacques Maritain (qui venait d'épouser une émigrée russe), le slaviste Paul Boyer, Rambaud... On peut distinguer ici trois cercles principaux : professeurs de la Sorbonne lettres et enseignants ou élèves de l'Ecole normale supérieure ; faculté et académie de médecine ; faculté des sciences, enseignants et préparateurs.

Cette énumération permet aisément de reconnaître une forte présence d'anciens dreyfusards parmi ces défenseurs de la révolution russe. Clemenceau, Jaurès, Pressensé, Jean Psichari, Ferdinand Buisson, Seignobos, Anatole France, Octave Mirbeau, Quillard, Maeterlinck, Jules Renard, Mendès, Lucien Herr, Langevin, Perrin, Séailles, etc., autant de noms qui étaient apparus au premier plan de «l'Affaire » depuis 1898, et notamment autour de la Ligue des droits de l'Homme. Nombre d'entre eux avaient aussi fait partie des réseaux qui s'étaient déjà élevés contre les massacres d'Arméniens et de juifs et qui avaient soutenu la cause

16. Nous nous sommes servi, pour toute cette partie concernant les personnes, d'un ensemble de publications, telles que L'Humanité, L'Aurore, Le Courrier Européen, Le Radical, La Tribune russe, et l'ouvrage: Anatole France, Vers les temps meilleurs. "Trente ans de vie sociale », vol. II, 1905-1908, P. : Émile-Paul, 1953 (commenté par Claude Aveline et Henriette Psichari). Ce travail est loin d'être exhaustif.

17. Voir Marie-Claire Bancquart, «Anatole France et la guerre russo-japonaise », in Dany Savelli, éd., Faits et imaginaires de la guerre russo-japonaise (1904-1905), P. : Kailash, 2005, p. 295-306, traduit en russe sous le titre : «Anatol' Frans i russko-japonskaja vojna : problesk nadeždy» [Anatole France et la guerre russo-japonaise: une lueur d'espoir], in O.R. Ajrapetov, red., Russko-japonskaja vojna 1904-1905. Vzgljad čerez stoletie [La guerre russo-japonaise, vue cent ans après], M. : « Tri kvadrata », 2004, p. 570-578. 
polonaise, ainsi Quillard et le réseau de Pro Armenia ${ }^{18}$ et, par conséquent, l'opinion de gauche française s'intéressait déjà depuis le tout début du siècle aux «crises russes ». Tout se passe donc comme si cette opinion et ces acteurs étaient restés « mobilisés » depuis l'Affaire Dreyfus jusqu'à la révolution de 1905 qui leur offrait beaucoup de blé à moudre dans la défense des opprimés.

Parmi ces défenseurs de la révolution russe, certains originaires de l'Empire furent particulièrement actifs. On connaît le rôle de Lavrov (mort en 1900 à Paris et toujours célébré) au sein de ces réseaux qui reliaient les Français d'une part et des émigrés politiques de l'Empire russe d'autre part. Souvent, comme on l'a vu, ces réseaux s'étaient déjà noués, par suite de contacts entre socialistes, ou encore à la faveur de campagnes pour la défense des Polonais, des Arméniens, des juifs de l'Empire. Dans ce domaine assez peu exploré, certaines personnes mériteraient qu'on s'y intéresse de plus près. Nous en citerons quelques-unes sans que, bien sûr, cette liste soit exhaustive. Rubanovič, d'abord, le plus connu, qui publia La Tribune russe en 1904-1905, puis de nouveau en 1909-1910 et qui alla de meeting en meeting en 1905-1906. Gor'kij (Gorki) aussi, bien sûr, plus accessoirement, qui publia dans L'Humanité, et dont la défense fut un des chevaux de bataille de la gauche européenne. Le constitutionnel-démocrate Evgenij Smenov (Eugène Semenoff), souvent invité dans les meetings, lié à Gor'kij dont il était traducteur. Petr Struve, membre de la rédaction de la revue L'Européen qui faisait suite au Courrier européen par suite d'une scission (en même temps que Pressensé, Anatole France, Seignobos, Vandervelde et le prix Nobel norvégien Björnstjerne Björnson), une revue très représentative de cette gauche républicaine anti-tsariste, et dans les colonnes de laquelle on retrouve Seignobos, Jaurès (avec lequel Struve dialogua dans L'Humanité du 8 juin 1905), et aussi le libéral Leroy-Beaulieu. Struve semblait aussi très lié à Lévy-Bruhl, ancien dreyfusard. Il faut très probablement citer Miljukov, bien que nous n'ayons aucune preuve directe de ses liens avec des historiens comme Aulard et Seignobos en 1905-190619. Ces deux derniers, avec quelques autres, adressèrent du reste une lettre de soutien à Kareev, professeur d'histoire à Sain-Pétersbourg et libéral notoire ${ }^{20}$. Enfin, nous nous arrêterons sur Stanislas Posner, Polonais dont on sait peu de chose (l'Okhrana, qui s'y intéressa, renonça à l'identifier), mais qui publia de 1905 à 1908 (?) La correspondance russe (Paris, Londres, Berlin), bulletin qui informait régulièrement sur les mouvements révolutionnaires russes, des cadets aux socialistes, et qui attaquait non moins régulièrement la politique russe du gouvernement français. Posner, qui était visiblement

18. A ce sujet, voir Edmond Khayadjian, Archag Tchobanian et le mouvement arménophile en France, Marseille : Centre régional de documentation pédagogique, 1986.

19. Il n'est pas sans intérêt de signaler que le manuel de Seignobos et Langlois fut publié en russe en 1899, l'ouvrage de méthodologie de Seignobos en 1902, son Histoire politique de l'Europe contemporaine en 1897, plus tard son Histoire du mouvement de libération en Russie. Quant à Aulard, son Histoire politique de la révolution française fut publiée en russe en 1902, De la monarchie à la république en 1906, Les orateurs de la révolution en 1907-1908.

20. Voir L'Humanité, 28 janvier 1905. 
très lié à Miljukov, avait correspondu avec l'historien russe cadet Kareev²1 et ses sympathies allaient à l'évidence au parti cadet. Nous avons la preuve indirecte qu'il était lié aux rédactions du Courrier européen et de L'Européen. Deux événements provoquèrent de vives réactions de ces revues: la dissolution de la Première Douma en juillet 1906 et le coup d'Etat de Stolypin en juin 1907. Après ce dernier, Posner, s'inspirant d'une pratique courante à l'époque et en puisant dans le carnet d'adresses de L'Européen, lança, visiblement avec l'aide de Miljukov, une enquête auprès de personnalités politiques et intellectuelles françaises et étrangères pour recueillir leurs avis sur ce qui venait de se passer $^{22}$.

Qu'avaient donc en commun les réactions de cette gauche dont nous avons esquissé les contours ? Passons sur l'anti-tsarisme, la stigmatisation du régime autocratique sur lequel tous se rejoignaient et qui était traditionnel dans la gauche française depuis 1848: en 1905-1906, au moment d'une forte tension francogermanique sur la question du Maroc, l'image du despotisme russe était en quelque sorte épaulée par celle de la monarchie allemande et brouillait les représentations optimistes de l'alliance.

Ce tsarisme était doté de ses symboles : Nicolas II, bien sûr, mais aussi Pobedonoscev, bête noire des libéraux et des socialistes russes. En France et pour beaucoup, il n'était pas seulement une incarnation de la réaction, il présentait aussi l'avantage d'incarner le cléricalisme ${ }^{23}$, de sorte que les affrontements russes pouvaient se lire à travers les questions qui agitaient la France au lendemain de la loi de la séparation. Stolypin n'avait guère meilleure presse du côté de la gauche, y compris dans Le Matin.

Les références à la Révolution française étaient très fréquentes, y compris dans le camp socialiste, si fréquentes qu'il ne serait pas exagéré d'affirmer que tout le déroulement de la révolution russe fut «lu », en France, à travers des lentilles fabriquées de 1789 à 1799, comme nous en avons déjà montré maints exemples. Louis XVI devenait naturellement Nicolas II. Turgot prenait, souvent à son désavantage, les habits de Witte. Les congrès des zemstva rappelaient les États-Généraux ${ }^{24}$, qui servaient aussi à caractériser la Première, voire la Deuxième Douma. Leroy-Beaulieu comparait l'effarement du chambellan de Louis XVI devant les députés du tiers état aux sentiments que devait éprouver le maître des cérémonies du tsar devant «les lourdes bottes des députés moujiks ${ }^{25}$. L'historien de 89 Aulard consacrait tout un éditorial dans L'Aurore du 28 mai 1906 à ce « rapprochement historique » entre les deux révo-

21. Lettre de Posner à Kareev s.d., Bibliothèque d'État de Russie (RGB, Moscou), Département des manuscrits, Fonds Kareev, carton 21, n 19. En 1917, Posner correspondit avec Joseph Reinach à propos de Lenin (lettre du 10 mai 1917, B.N., ms., NAF 13553,f. 171-171v.)

22. Nous comptons publier ultérieurement une étude de cette enquête inédite.

23. Alexandre Ular dans L'Aurore du $1^{\text {er }}$ janvier 1905, à propos de Nicolas II : « [...] et il compte, avec son médiéval Pobiédonostseff, que le Dieu qui l'a institué autocrate, saura défendre son œuvre $[\ldots] »$

24. « Rien de nouveau sous le soleil », écrit Clemenceau dans l'Aurore du 27 juillet 1905, dans un éditorial intitulé « Les États-Généraux à Moscou » à propos du second congrès des zemstva.

25. « La Douma et la révolution russe », Journal des débats, 7 juin 1906. 
lutions. Seignobos, autre historien de la France et de l'Europe, écrivait : « La ressemblance est si frappante qu'elle commence à devenir banale. » Et de comparer les deux révolutions terme à terme: les philosophes français devenaient les intellectuels russes, les parlements se muaient en zemstva ${ }^{26}$. Même lorsque les pronostics étaient prudents, le parallèle allait de soi, et surtout, chacun s'accordait généralement pour défendre l'idée que la Russie avait son tiers état (ce fut l'objet d'un article de Gaston Leroux dans Le Matin du 4 novembre 1905) et que ce tiers état allait supplanter inéluctablement le régime absolu en imposant une forme de gouvernement représentatif ${ }^{27}$. Certains (par exemple, Clemenceau, la presse du centre, Paul Boyer dans une interview ${ }^{28}$ ) craignaient la masse paysanne russe, mais ce peuple était moins « écrasé par l'ignorance et [...] abêti par le despotisme » qu'on ne le supposait ${ }^{29}$. Par conséquent, tous se rejoignaient pour soutenir la représentation nationale russe, au point Clemenceau comme on l'a vu, Seignobos dans un article publié dans L'Européen du 18 février 1905 - de vouloir soumettre le nouvel emprunt russe à l'approbation, et donc au contrôle budgétaire, de la nouvelle assemblée.

L'opinion républicaine se caractérisait ainsi par un continuum d'opinions qui, tout en scrutant la révolution russe, fondait libéralisme et socialisme, révolution politique et révolution sociale dans une perspective éminemment française, en laquelle 1789 rejoignait le temps présent en passant par 1848, ainsi qu'on peut le lire dans ce raccourci très expressif de Francis de Pressensé : «Entre la terre des droits de l'homme et la Russie libérale et socialiste, prolétaire et constitutionnelle, entre elles seules il y a un pacte valable, un pacte plus fort que les grimoires de la diplomatie. Le tsarisme n'est qu'un accident. $»^{30}$

Or ce continuum que nous avons observé entre les socialistes et l'opinion républicaine en France se retrouvait en Russie, du moins en façade, par exemple dans La Tribune russe ou La Correspondance russe, entre socialistes et cadets, dès lors qu'il s'agissait de crier « À bas l'autocratie » : c'était le camp de la révolution. Mais la symétrie n'était qu'apparente. Si les socialistes russes trouvaient sans grande peine leurs interlocuteurs chez leurs homologues français, les cadets russes éprouvaient davantage de difficulté, car ils se heurtaient à la raison d'État, autrement dit à l'alliance franco-russe, sitôt qu'ils visaient le parti radical ou à plus forte raison le gouvernement français. Certes, avec l'aide d'Anatole France et de Pierre Quillard, des dirigeants du parti cadet comme Maklakov et Pavel Dolgorukov réussirent à approcher Clemenceau, président du conseil, et d'autres personnalités « républicaines » comme Raymond Poincaré afin que le gouvernement français

26. Le Courrier européen, 18 février 1905.

27. « La Russie, comme toute l'humanité, marche à l'inévitable, et l'inévitable, c'est le droit, la justice, la liberté » (Clemenceau, dans L'Aurore du 21 décembre 1904).

28. « Le mouvement social en Russie », L'Aurore, 2 janvier 1905.

29. La Lanterne, 6 janvier 1905.

30. L'Humanité, 29 janvier 1905. Au sujet de Francis de Pressensé, voir sa biographie par Rémi Fabre, Francis de Pressensé et la défense des droits de l'homme. Un intellectuel au combat, Rennes : Presses Universitaires de rennes, 2004, notamment p. 350-358 sur la révolution russe. 
entourât de conditions politiques l'émission de l'emprunt d'avril 1906. Sans succès $^{31}$. C'est pourquoi les cadets allaient souvent chercher leurs sympathisants plus à gauche que leurs cousins naturels, les radicaux. Semenov, déjà cité, pouvait publier dans $L^{\prime}$ Humanite ${ }^{32}$. Seuls les professeurs républicains français répondaient entièrement à leur attente puisqu'ils alliaient leur libéralisme politique à l'intransigeance de leur condamnation de l'autocratie, partageant entièrement avec les cadets le modèle de 1789 .

CERCEC EHESS, Paris, Université de Genève

Wladimir.Berelowitch@ehess.fr

31. À ce sujet, voir la thèse inédite de James William Long, The Economics of the FrancoRussian Alliance, 1904-1906, op. cit., p. 210-213.

32. Le 11 avril 1906, pour annoncer que les Cadets refuseraient de ratifier l'emprunt de 1906. 